\title{
Will mHealth Revolutionize Health and Clinical Management and Open up New Horizons for Mental Health?
}

\author{
E. Conchon', N. Bricon-Souf ${ }^{2}$, Section Editors for the IMIA Yearbook Section on Health and \\ Clinical Management \\ I University of Limoges, XLIM, UMR 7252, Limoges, France \\ 2 University of Toulouse, IRIT, Castres, France
}

\section{Summary}

Objectives: To summarize recent research and emerging trends in the field of Health and Clinical management and propose a selection of best papers for year 2015.

Methods: A literature review has been conducted by the two section editors and computerized provider order entry systems from bibliographic databases regards to health clinical management. As a result, a list of 15 candidate papers has been elaborated and a peer-reviewed has been performed by external reviewers. A consensus meeting has been organized between the two section editors and the editorial board to conclude the selection of the 3 best papers.

Results: Starting with 1803 papers published in 2015, the full selection process ended with three papers from international peer-reviewed journals for the Health and Clinical Management section.

Conclusion: I0T and Cloudification have a direct impact on health and clinical management this year. Telepsychiatry benefits directly from this development and take advantages of the improvement of smart homes and of the generalization of mHealth solutions. Social networks are starting to be integrated as valuable source of information that are complementary to clinical data for reasoning-based solutions.

\section{Keywords}

Health and Clinical Management; patient care management; medical record systems; technologies in healthcare; telemedicine

Yearb Med Inform 2016:109-12

http://dx.doi.org/10.15265/IY-2016-046

Published online November 10, 2016

\section{Introduction}

Originally focused on institutional information systems and cross-border uses of health data, the scope of the Health and Clinical Management section has been extended to cover Telemedicine, health applications of ubiquitous computing as well as Ambient Assisted Living. Based on a careful review of research papers published in 2015, a selection of three best papers that illustrate the research trends relevant for the Health and Clinical Management section is proposed.

As in previous years, many research works target telemedicine trials with a growing interest on the management of cancer and chronic diseases. But, as in the previous edition of the yearbook, it must be noted that most of these trials are focused on a single hospital or health facility so that no generic conclusion can be made. Specificity for this year is the diminishing number of telemedicine evaluations based on online questionnaires. Mobile applications as well as SMS seem to become the preeminent solution to have feedback from users at the expense of online surveys.

Last year, the importance of smart and efficient exploitation of data has been straightened [1]. This trend that emerged two years ago is still growing very quickly. Indeed, while the amount of data from a variety of sources increases, there is still a lack of analysis tools dedicated to health care management. It can be noticed that these tools will have to deal with very big numbers of heterogeneous data so that data mining solutions seem to be promising. To support this assertion, it is interesting to note that a large number of papers published this year are relying on text mining or data mining over health information system but also over social networks.

The cloudification of health data that has been pointed out last year is still very important with the emergence of m-health as a preferred way to gather health data as well as additional information (e.g. location). For instance, lots of m-health solutions have been proposed during this year to deal with diabetes, obesity or depression. In order to go further on this topic, the HCM section survey is reviewing eHealth and mHealth solutions for cancer and chronic disease management.

\section{About the Paper Selection}

A database search has been performed on Medline for papers published in 2015 that are relevant with Health and Clinical Management. As in the previous year, mobile health applications, ambient assisted living solutions and telemedicine have been considered in addition to more classical terms such as health clinical record, care management, database management or Health Information System. This database search has returned a total of 1803 papers after excluding papers without abstracts or authors. Each section editor has reviewed half of the papers in order to keep about 100 papers each. The selections have then been 
merged and the remaining 200 papers have been reviewed by both editors. At the end of this step only 30 papers per editor remain. For this selection, the editors have preferred innovative papers rather than surveys and have only kept trials that have a large number of patients involved, countrywide evaluation or cross-borders considerations. A formal meeting has been set up so that editors can finally propose a list of $15 \mathrm{pa}$ pers to external reviewers (at least four per paper). Three papers were finally selected as 2015 best paper for Health and Clinical Management section. A short description of these papers is provided in the appendix of this synopsis.

\section{Discussion and Outlook}

The first paper by Akl et al. presents an autonomous system to detect mild cognitive impairments in an ageing population based on information gathered by environmental sensors in smart homes and on clinical data. This article underlines the interest to integrate several sources of information as well as the impact of smart homes to detect the deterioration of health status. Indeed, the recent improvements in smart homes as depicted in [2] enable more and more possibilities for the detection of activities of daily living that can be then correlated with clinical data to improve the medical follow-up of patients by health practitioners. Akl et al. paper is also interesting due to the number of patients involved in the evaluation (97). In previous years most evaluations were limited to a few numbers of smart homes and patients thus limiting their impact and the conclusion that can be made from a clinical standpoint.

The second best paper written by Lanata et al. points out the significant progresses that have been made to collect medical data with a focus on mental health management. Mental health is of major interest in health and clinical management since few years with a growing number of papers on dedicated systems for clinical applications or telemedicine projects that aim to provide follow-ups for patients with psychiatric disorders. Lanata et al. propose an innovative

Table 1 Best paper selection of articles for the IMIA Yearbook of Medical Informatics 2016 in the section 'Health and Clinical Management'. The articles are listed in alphabetical order of the first author's surname.

\section{Section}

Health and Clincal Management

- Akl A, Taati B, Mihailidis A. Autonomous unobtrusive detection of mild cognitive impairment in older adults. IEEE Trans Biomed Eng 2015;62(5):1383-94.

- Lanata A, Valenza G, Nardelli M, Gentili C, Scilingo EP. Complexity index from a personalized wearable monitoring system for assessing remission in mental health. IEEE J Biomed Health Inform 2014;19(1):132-9.

- Simplicio MA, Iwaya LH, Barros BM, Carvalho TC, Näslund M. SecourHealth: a delay-tolerant security framework for mobile health data collection. IEEE J Biomed Health Inform 2015 Mar; 19(2):761-72.

system to help bipolar patients by detecting their mood states based on a wearable system coupled with biomedical signal processing and data mining technics.

The third best paper selected is complementary to the two others as it proposes a delay-tolerant security framework to collect data from mobile devices. This article written by Simplicio et al. highlights the need of security in mobile health data collection and proposes several protocols to achieve that. The security topic is still very active in Health and Clinical Management section as several dedicated solutions or protocols have come up from the Medline database search. For instance, Zaidan et al. [3] have proposed a nation-wide security framework for the exchange of health information that mainly relies on existing security solutions and is adapted to the Malaysian health system.

Several other papers published in 2015 deserve to be cited. They address more traditional topics that are still very active in the framework of the section. For instance, Sarker et al. [4] propose an adverse drug events detection system based on automatic text classification. The originality of this system lies in its ability to dig in social media for medical information in order to improve the detection of adverse drug events. Social media are getting an increasing attention from researchers dealing with health and clinical managements making their integration in health information systems an important trend to follow in the upcoming years. On a similar topic, a system such as MERIS proposed by Riga et al. [5] is trying to cope with adverse events and proposes new innovative solutions that are promising but have to be evaluated on a broader scale. The authors point out that the lack of political decision about healthcare policy is the main factor preventing a generalization of such systems. These questions of cross-border and nation-wide standardizations are still open issues to help the development of relevant tools for health and clinical management. Park et al. [6] present the approach retained by New Zealand to address this issue and a comparative study with other countries could be of major interest. Finally, and as expected in the past few years, mHealth is becoming the new game changer for health. Indeed, mHealth is starting to replace the use of web applications and surveys for patients' follow-up. Moreover, numerous health solutions based on smartphones have been proposed during 2015 as shown by Silva et al. [7]. But, these solutions have to be evaluated over the long term and on larger numbers of patients to measure their efficiency.

\section{Acknowledgement}

We would like to acknowledge the support of Martina Hutter and of the reviewers in the selection process of the IMIA Yearbook.

\section{References}

1. Bricon-Souf N, Conchon E. A 2015 Medical Informatics Perspective on Health and Clinical Management: will cloud and prioritization solutions be the future of health data management? Yearb Med Inform 2015;44-6.

2. Güttler J, Georgoulas C, Linner T, Bock T. Towards a future robotic home environment: a survey. Gerontology 2015;61(3):268-80.

3. Zaidan BB, Haiqi A, Zaidan AA, Abdulnabi M, Kiah ML, Muzamel H. A security framework for nationwide health information exchange based on telehealth strategy. J Med Syst 2015;39(5):51.

4. Sarker A, Gonzalez G. Portable automatic text 
classification for adverse drug reaction detection via multi-corpus training. J Biomed Inform 2015;53:196-207.

5. Riga M, Vozikis A, Pollalis Y, Souliotis K. MERIS (Medical Error Reporting Information System) as an innovative patient safety intervention: a health policy perspective. Health Policy 2015;119(4):539-48.

6. Park YT, Atalag K. Current National Approach to Healthcare ICT Standardization: Focus on Progress in New Zealand. Healthc Inform Res 2015;21(3):144-51.

7. Silva BM, Rodrigues JJ, de la Torre Díez I, López-Coronado M, Saleem K. Mobile-health: A review of current state in 2015. J Biomed Inform 2015;56:265-72.

\section{Correspondence to:}

Emmanuel Conchon

XLIM

University of Limoges

123 Avenue Albert Thomas

87000 Limoges, France

E-mail: emmanuel.conchon@xlim.fr

\section{Appendix: Content Summa- ries of the Selected Best Pa- pers for the IMIA Yearbook 2016, Section "Health and Clinical Management"}

\author{
Lanata A, Valenza G, Nardelli M, Gentili C, \\ Scilingo EP \\ Complexity index from a personalized \\ wearable monitoring system for assessing \\ remission in mental health
}

\section{IEEE J Biomed Health Inform $2014 ; 19(1): 132-9$}

This research focuses on the monitoring of patient suffering from bipolar disorder. This psychiatric disease is frequent, altering mood regulation while balancing patient's state from depression to hypomania. It induces high managing cost, disease management and treatment are difficult and disease diagnoses are complicated. To perform the diagnoses or the effects of the treatments, psychology questionnaires are usually used and interpretation relies on subjective data.

This research explores how a wearable system could help to improve the evaluation of the patient's state in order to better calibrate the care. A wearable system called the PSYCHE system proposes embedded captors into a sensored t-shirt connected to a smartphone via Bluetooth for the patient. Physicians are proposed a visualization platform to support clinical decision.

In order to discriminate different mood states from autonomic nervous system signals and in particular circadian heart rate rhythms, data acquired from captors are preprocessed and treated through data-mining. Other signals such as biochemical data could be used by such a system. In the context of this study, a signal named SampEn for sample entropy, based on the measure of heart rate variability, is proposed in order to provide an information on the evolution on mood state.

An experimental protocol has been proposed: ten hospitalized patients suffering from bipolar disease were equipped with the PSYCHE system and followed until remission; they were regularly diagnosed during their stays and their mood state was classified as euthymia state, depression state or mixed state. The SampEn signal was then calculated and appears as an effective measure of the treatment response of each patient toward remission, in particular this entropy signal varies linearly when pathological state goes toward euthymia.

\section{Akl A, Taati B, Mihailidis A}

\section{Autonomous unobtrusive detection of mild cognitive impairment in older adults}

\section{IEEE Trans Biomed Eng 2015;62(5):1383-94}

With the growing percentage of people older than 65 in Canadian populations, facing Alzheimer disease or other dementia is important, moreover such diseases are often under-recognized. This study tackles this difficulty, proposing early detection process through unobstructive sensors and smart systems. Mild Cognitive Impairment (MCI) has been showed related to the slowing of walking speed.

The study involved 97 homes equipped with unobstructive sensing technologies; sensors data as well as clinical ones were collected over 3 years. Many other smarthome projects exist in the literature but most of them are laboratory experiments and not real life ones. Research questions were on the possibility of detecting MCI from the sensing technologies, on the necessary span time and how the measured features impact the MCI detection.

By using weekly walking speed and general activities in home, the authors shown that a signal processing approach with machine learning can be used to discriminate older adults with $\mathrm{MCI}$, a period of 24 weeks gives good results, measures and features were ranked in term of MCI detection ability and finally the effect of real world with noisy data.

A dataset of weekly measures vectors was used; sliding windows allow to concatenate the data in order to use machine learning algorithms. Sensitivity, specificity and precision were then studied according to the cognitive status recognition.

Subject's home were equipped with passive infrared motion sensors on rooms, contact switches on exit doors, ceiling motion sensors on hallway or corridors, able to wireless connect to a transceiver and a SQL database of firings was performed. In the same time, subjects reports on their weekly way of life (visitors, medication change).

Subjects were assessed annually through a cognitive score and labelled in cognitively, unknown or suffering from MCI category.

From the sensors data, different measures were computed concerning the activity, the walking, the period of the day for example. Among the 97 home, 68 remain while having data not too noisy. Measure vectors were labelled with cognitive status and fed the machine learning algorithms. SVM and RF machine learning algorithms were used to tackle the classification onto cognitive status recognition. Different features were tested and analyzed.

In order to discriminate older adults with MCI from non MCI ones, a time window of 24 months was preferred. Other important variables were found to be: trajectories of weekly walking speed, coefficient of variation of the walking speed, coefficient of the variation of the morning and evening walking speed, and subject's age and gender.

\section{Simplicio MA, Iwaya LH, Barros BM, Carvalho TC, Näslund M}


SecourHealth: a delay-tolerant security framework for mobile health data collection

\section{IEEE J Biomed Health Inform 2015 Mar; 19(2):761-72}

The authors provide a solution to deal with data collection in mHealth with a specific focus on security. The authors are interested in the way health care workers can be used to collect health data during the day. These workers are in charge of visiting families and of filling electronic forms to provide data to the health management system. This way of collecting data can be viewed as a delay tolerant network as the health care workers do not always have network connectivity. Moreover, as they have to collect sensitive and sometimes personal infor- mation, security is a key issue to consider.

The authors then propose SecourHealth a mHealth security framework for health data collection over delay tolerant networks. This framework offers several functionalities such as users' registrations, offline authentication of users, secure data storage and secure data exchanges between the mobile application and the server.

The registration process is made when a user first accesses to a new mobile terminal and relies on a new authentication protocol between the mobile phone and the online server. After a first successful authentication, a user can then perform offline authentication thanks to locally stored credentials. This step is very important in delay tolerant network architecture as users will not always have a full connectivity with the online server. The secure storage and theft avoidance are realized with three different encryption keys and a dedicated encryption algorithm that offers three security levels: no forward secrecy, weak forward secrecy and strong forward secrecy. Strong forward secrecy is the most secure mode where an attacker is not able to recover data even if he has an access to the original password or to the device memory.

Finally, a secure exchanged protocol between mobile phones and the online server is provided. A practical evaluation of the framework is then proposed. Indeed, SecourHealth has been integrated in the GeoHealth system, a governmental program used in Sao Paulo for family health program. 